\title{
Report of the Conference of Lepra Regional Organizing Secretaries
}

(held at Farnham, Surrey)

\author{
Kindly written by Dr S. G. BROWNE, O.B.E., M.D., F.R.G.s., D.T.M. \\ Medical Secretary, British Leprosy Relief Association, 8 Portman Street, London W.I., England
}

The British Leprosy Relief Association, now known as LEPRA (formerly BELRA), held a very successful residential conference for its Regional Organizing Secretaries at Farnham, Surrey, England, in May of this year (1966). Under the genial chairmanship of Sir George Seel, k.c.m.G., Chairman of Lepra, the participants not only considered questions of propaganda and organization and fund-raising, but also gave themselves as laymen to the study of leprosy as a major endemic disease in the countries of the Commonwealth.

Interesting factual reports of their recent visits to Africa were given by Mrs Peggy Morton, Regional Organizing Secretary for N.E. England, and by Mr Francis Harris, M.c., Deputy General Secretary of LEPRA.

Dr Gordon Currie, O.B.E., gave the conference up-to-date news concerning the Leprosy Control Project inaugurated recently by LEPRA in Malawi. In the chosen area of operations there are probably io,ooo people suffering from leprosy. Communications are quite good, and the people are courteous and friendly. The Project is assured of the personal interest and wholehearted co-operation of the President, Dr Hastings Banda. Dr Currie himself, who as Government Leprologist had an enormous fund of local knowledge and enjoyed the esteem of the people, directed the scheme in its initial stages. To the regret of all, circumstances have compelled Dr Currie to return to Britain. His place has been taken by Dr David Molesworth, formerly of Malaya and Ghana. He is assisted by two LEPRA field-workers, and will shortly be joined by an experienced nursing sister and a laboratory technologist.

Auxiliary staff are being trained on the spot in leprosy diagnosis and treatment, and rural surveys are under way. Already, many hundreds of leprosy patients have been brought under treatment.

It is hoped that expert surgical assistance will be available for the correction of deformities due to leprosy - all in keeping with the scope and objects of the Project, one of which is to demonstrate to countries with similar medical and financial problems a practical method of controlling and eventually of eradicating leprosy in a circumscribed area.

A small central hospital will be erected in the grounds of the Queen Elizabeth Hospital at Blantyre, where the laboratory, administrative headquarters, and rehabilitation unit will be installed.

Dr S. G. Browne, O.B.E., the new Medical Secretary of LEPRA, gave a stimulating address on 'Modern Leprosy Work'. He emphasized the need for hard work and adequate knowledge and perseverence in the struggle against leprosy. Gone were the days of easy optimism engendered by the advent of the sulphones, and by the popularization of orthopaedic surgery as applied to leprosy. Despite the numerous successes of the past decade, leprosy still constitutes a formidable medical, social and financial problem to those countries least able to afford or to face its ravages.

Leprosy work today entails bringing the known effective drugs to the people who need them, which in turn means discovering by surveys and examination of contacts all who are suffering from leprosy, and making available the standard drugs to them through static or mobile treatment centres. Dr Browne emphasized the importance of diagnosing leprosy early, diagnosing it correctly and placing patients under adequate treatment before irreversible nerve damage had occurred. 
Secondly, leprosy work means training indigenous auxiliary workers in leprosy diagnosis and control, in surveys, in examinations of contacts, in simple microscopy and simple physiotherapy, in mass treatment. The role of these invaluable auxiliaries was to cure the individual suffering from leprosy, to prevent nerve and eye damage, to educate patients and their entourage, to supply simple footwear that will prevent neuropathic ulceration, and to ensure that patients under treatment or with arrested disease may retain or resume their place in the family and the community.

Thirdly, modern leprosy work includes the provision of a central hospital where patients with special needs may receive expert help. The over-emphasis on mass treatment in the immediate past must be counterbalanced by insisting on the need for a well-equipped and well-staffed centre where a variety of patients may be treated: those in acute exacerbation, or with incipient nerve or eye damage; those in drug reaction or needing stabilization of dosage; and those needing operation, physiotherapy, special footwear or prostheses.

Fourthly, leprosy work means education education of leprosy workers themselves (doctors, nurses, physiotherapists, etc.), and of medical auxiliaries of all kinds. It means education of the patients, so that they may look after themselves and prevent the many avoidable disabilities. Thus it means educating the community by all means and at all levels, especially in the preventive aspects of leprosy.

Fif thly, leprosy work today necessarily includes research into leprosy. New approaches and new investigative techniques have recently become available. New drugs are urgently needed, to reduce the unconscionably long period now necessary for treatment, to facilitate the removal of effete mycobacteria, and to minimize the over-vigorous immunological response of the presence of mycobacterial antigen. The many and serious lacunae in our knowledge of Myco. leprae must be filled in as soon as may be.

Sixthly, modern leprosy work visualizes more than ever before the need for co-operation between all those engaged in the struggle. The Christian missions that have been in the forefront of the fight are now joined by non- confessional bodies, by Governments and by international agencies.

Lastly, it may be possible within the comparatively near future to make a definite pronouncement concerning the prophylactic value of B.C.G. inoculation in leprosy. If the present encouraging results are confirmed in the final reports from Uganda and elsewhere, there will be available a method for preventing the appearance of leprosy infection in three-quarters of the numbers of those exposed children who would otherwise contract it. If this happy result should prove to be true, then real and definite and lasting hope would arise in individuals and in countries where leprosy is still a scourge, an unresolved problem, and an economic incubus.

Mr W. Lennox, F.R.c.s., the orthopaedic surgeon who has been recently working inVellore, South India, and at the Karigiri Leprosy Research Centre sponsored jointly by the Leprosy Mission and the American Leprosy Missions, Inc., gave an account, illustrated by coloured transparencies, of the complex activities of a Rehabilitation Unit.

He showed the various types of severe damage that may result from repeated neglected trauma sustained in the course of ordinary walking, walking over irregular ground, and working with the hands. When a specific action can be incriminated as causing damage to an anaesthetic part, appropriate steps to mitigate or avoid its effects could and should be taken.

The surgeon's task comprises not only the accurate and detailed assessment of the departure from the normal, but also an appreciation of the cause, and the determination of the part to be played by each member of the team concerned in the total rehabilitation of the patient. The actual operation was but an incident in this long process.

When deformity has been corrected by operation and physiotherapy, the task of ten remains of making the patient cosmetically acceptable to his community. Thus, where time and opportunity exist, plastic and reparative operations while not strictly and surgically imperative may make all the difference to a patient's life and welfare.

The skills of the shoe-maker and splint-maker are also enlisted so as to provide the patient with 
shoes and splints to enable him to take his full place in the community. Tools and implements with specially made handles are often needed.

The psychological aspects of rehabilitation were also stressed, and the need to educate the patient to rethink his attitude to the disease and to any remaining disability he has. And then, prevention of recurrence is all-important, or the last state of the operated patient (who still has no tactile sensation in his extremities) may be worse than the first.

When asked what he considered the place of surgery in such a scheme as the Malawi Eradication Project, Mr Lennox admitted that early diagnosis of leprosy and adequate treatment would make virtually all reparative surgery unnecessary. If resources are limited, then money should be spent on bread, and not on the icing of a cake. 